\title{
Cumulative Evidence for Association of Acetaminophen Exposure and Allergic Rhinitis
}

\author{
Yan Zeng ${ }^{\mathrm{a}}$ Bin Song ${ }^{\mathrm{b}}$ Yang Gao ${ }^{\mathrm{a}}$ Wen $\mathrm{Cao}^{\mathrm{b}}$ Jia $\mathrm{Li}^{\mathrm{a}}$ Qianfang $\mathrm{Liu}^{\mathrm{a}}$ \\ Jian Liu $^{\text {a }}$ \\ a Department of Pediatrics, People's Hospital of Deyang City, Affiliated Hospital of Chengdu Medical College, \\ Deyang, China; bepartment of Nephrology, People's Hospital of Deyang City, Affiliated Hospital of Chengdu \\ Medical College, Deyang, China
}

\section{Keywords}

Acetaminophen · Allergic rhinitis · Meta-analysis · Risk factors

\begin{abstract}
Background: Several studies have linked acetaminophen exposure to the risk of allergic rhinitis, with controversial results. Methods: A systematic search of PubMed, Embase, Cochrane Library, and Web of Science was conducted up to December 1,2019 . We included observational studies that examined the association between acetaminophen exposure and the risk of allergic rhinitis. Two reviewers independently extracted data and assessed study quality. Summary odds ratios (ORs) and 95\% confidence intervals (Cls) were calculated using randomeffects meta-analysis. Results: Eighteen studies with 870,492 participants were included. Analysis showed that acetaminophen exposure was associated with an increased risk of allergic rhinitis (OR: 1.54, 95\% Cl: 1.41-1.69). Also, early exposure and exposure in the past year to acetaminophen was positively associated with risk of allergic rhinitis (OR: $1.34,95 \% \mathrm{Cl}$ : 1.21-1.49 and OR: $1.74,95 \% \mathrm{Cl}: 1.48-2.04$, respectively). The risk of allergic rhinitis was greater for people who had acet-
\end{abstract}

aminophen exposure once per month over the past year (OR: 1.90, 95\% Cl: 1.60-2.26) compared to once per year (OR: 1.28, 95\% Cl: 1.11-1.48). Conclusion: With our meta-analysis, we provide the evidence that exposure to acetaminophen is associated with an increased risk of allergic rhinitis. In addition, acetaminophen exposure in early life and acetaminophen exposure in the past year are positively associated with the risk of allergic rhinitis. Future research is needed to evaluate whether the association is causal.

(c) 2020 S. Karger AG, Base

\section{Introduction}

Allergic rhinitis is estimated to affect $10-30 \%$ of the population in the United States and other industrialized countries [1-5]. It is associated with a huge economic burden, causing a total cost of several billion dollars per year [6-8]. Although genetic factors play an important role in susceptibility to allergic rhinitis [9], environmen-

Edited by: H.-U. Simon, Bern.

karger@karger.com

www.karger.com/iaa

Karger
(C) 2020 S. Karger AG, Basel

. 


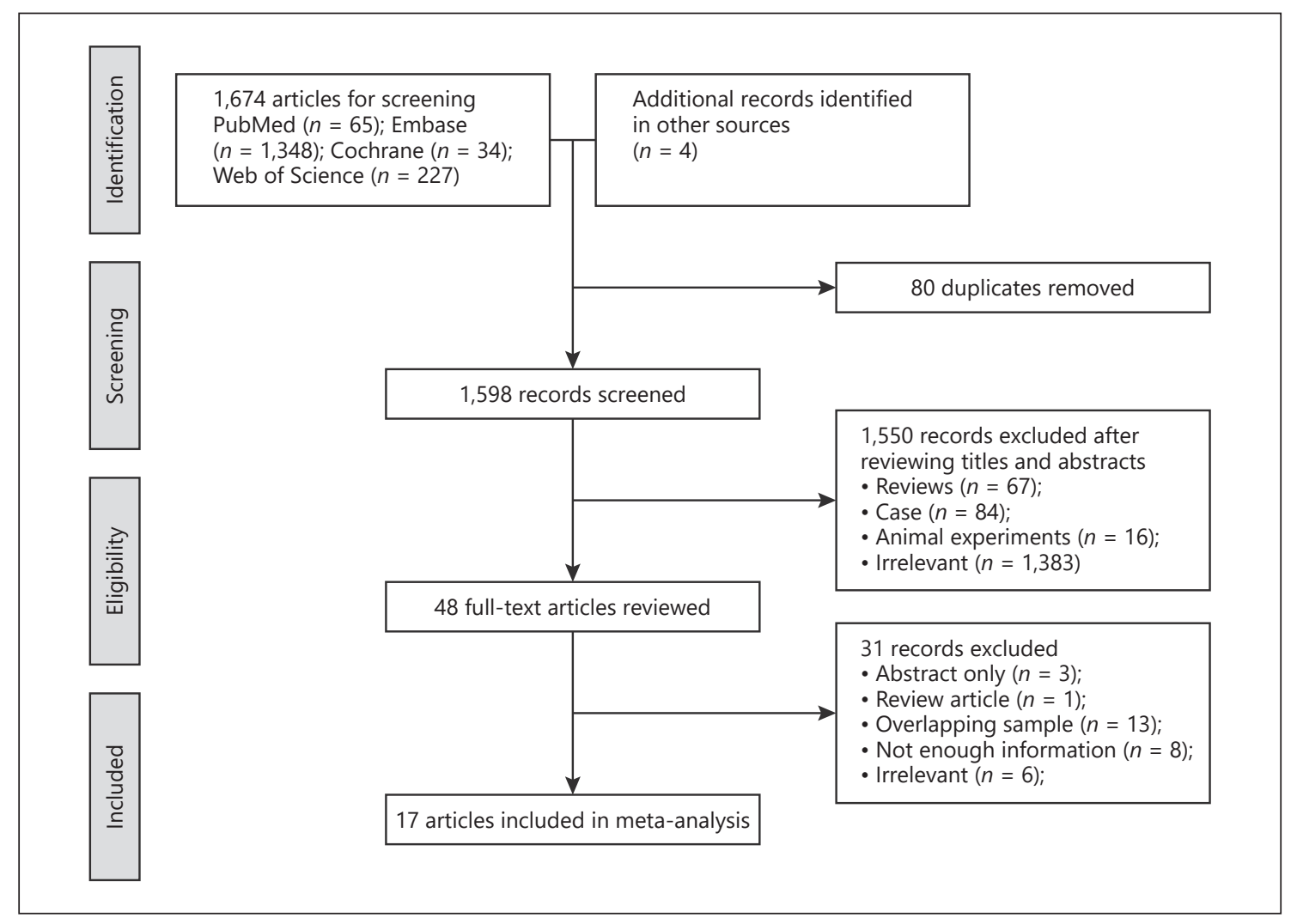

Fig. 1. Flowchart of study inclusion and exclusion process.

tal factors might underlie the increased prevalence [10]. The etiological factors influencing the development of allergic rhinitis remain uncertain.

Acetaminophen, which is generally considered safe, has become the most widely used analgesic antipyretic drug [11]. Recent studies have indicated a possible connection between acetaminophen use and allergic rhinitis. In one study, the increased sales of acetaminophen were associated with the rise in the prevalence of allergic rhinitis in children and adults [12]. In the large, multicenter International Study of Asthma and Allergy in Childhood (ISAAC) phase III, results showed that acetaminophen exposure represents a risk factor for allergic rhinitis in the 6- to 7-year-old and 13-to 14-year-old age groups [13, 14]. Consistent with these findings, several studies found associations between acetaminophen use and risk of allergic rhinitis [15-18]. However, some results are inconsistent $[19,20]$. Given the widespread use of acetaminophen and the importance of clarifying the role of its use in the risk of allergic rhinitis, we conducted a meta-analysis with aims to evaluate the association between acetaminophen exposure and risk of allergic rhinitis.

Meta-Analysis of Acetaminophen and Allergic Rhinitis

\section{Methods}

\section{Search Strategy}

We performed this meta-analysis in accordance with the Metaanalysis of Observational Studies in Epidemiology (MOOSE) guidelines [21]. A systematic literature search was conducted in PubMed, Embase, Web of Science, and Cochrane Library databases from inception to December 1, 2019. The following search terms, including Medical Subject Heading terms and free-text terms, were used in search: ("Acetaminophen" OR "Acetominophen" OR "Paracetamol" OR "Hydroxyacetanilide") AND ("Rhinitis, Allergic” OR “Allergic Rhinitis” OR “Allergic Rhinitides” OR “Allergic Rhinosinusitis" OR “Allergic Rhinitis”). The search was limited to English-language studies. In addition, the reference lists and citations of included studies were searched for relevant articles.

\section{Study Selection}

Two reviewers (Y. Gao and W. Cao) independently scrutinized the searches. They scanned the titles and abstracts of all articles to exclude irrelevant studies. Full texts of the remaining articles were reviewed to assess which articles met our inclusion criteria. Articles were considered eligible for inclusion in the study when they met the following criteria: (1) cohort, case-control, or cross-sectional studies that examined the association between acetaminophen exposure and allergic rhinitis, (2) odds ratios (ORs), risk estimates, risk ratios, or hazards ratios, and the corresponding 95\% confidence in- 


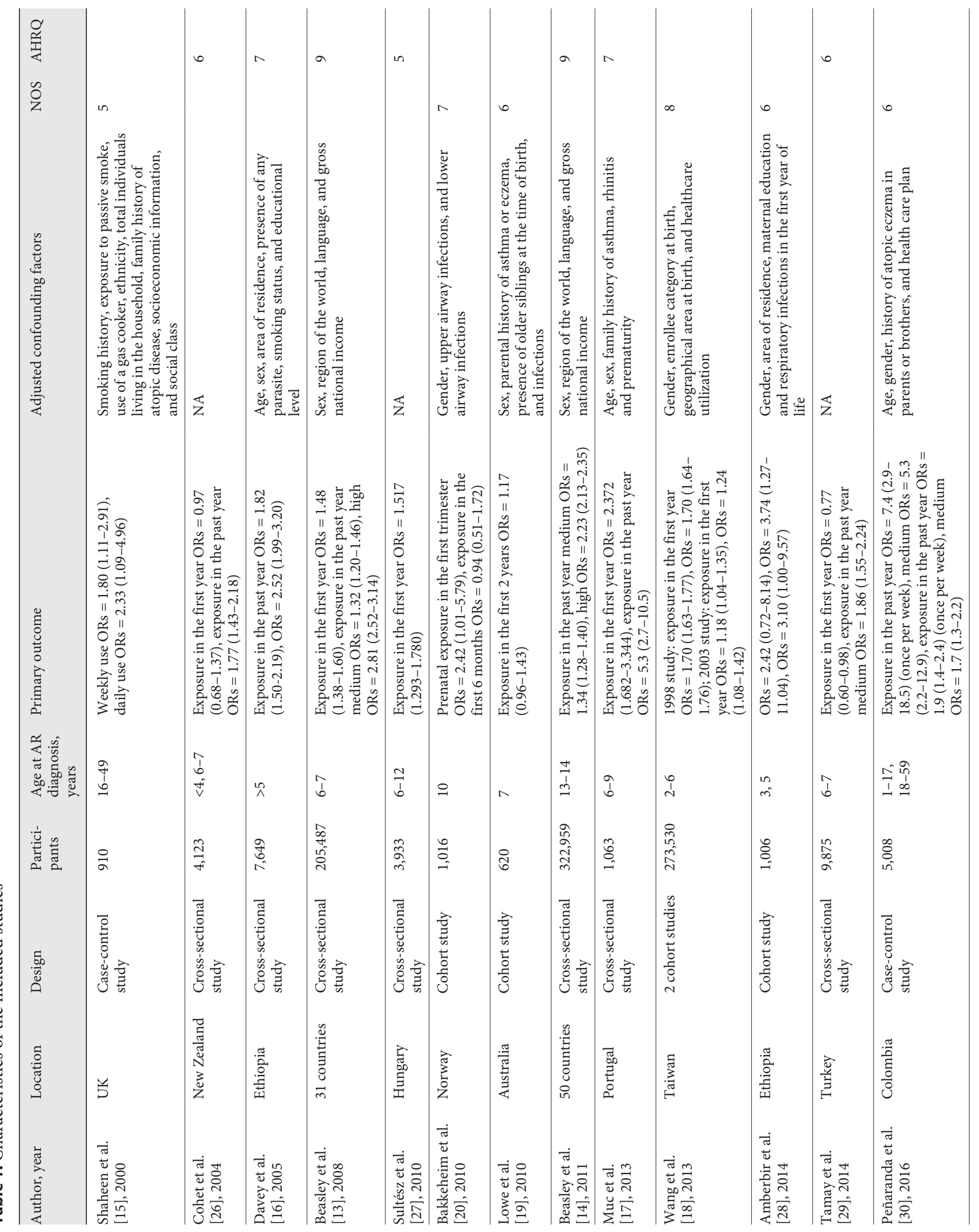



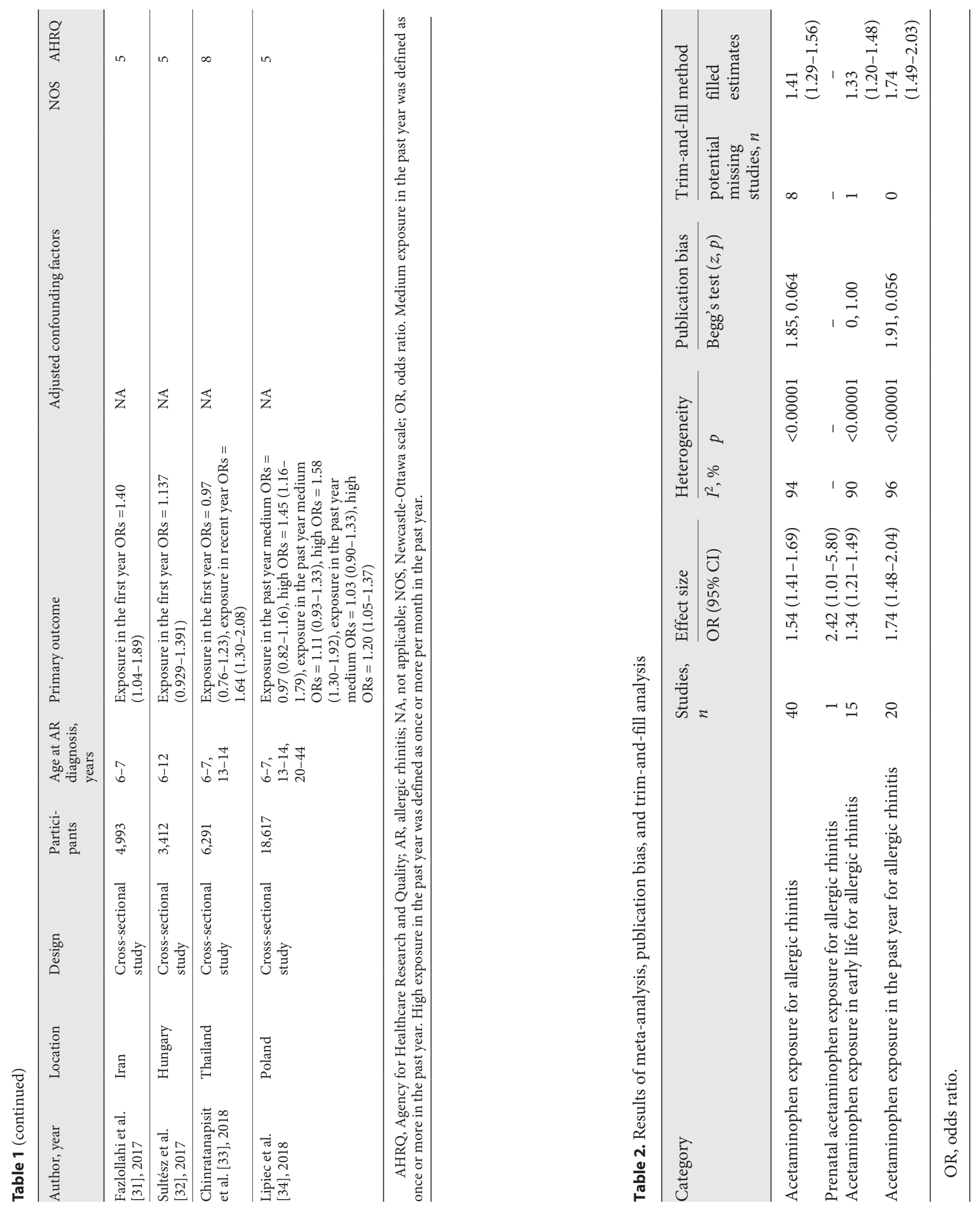
tervals (CIs) were reported, and (3) data to calculate the effect size was provided. Articles were excluded if they were: (1) reviews, letters, case reports, or animal research, or (2) lacking a comparison group. Any discrepancies between the two reviewers were discussed with a third reviewer (Q. Liu) to achieve a consensus.

\section{Data Extraction}

Two reviewers (Y. Gao and W. Cao) independently extracted the following information from the eligible studies: first author, year of publication, location, study design, study size, age of allergic rhinitis diagnosis, adjusted confounding factors, and outcome parameters with corresponding 95\% CIs. The acetaminophen exposure was classified into 4 categories: prenatal, early life, the past year, and other. Exposure in early life was defined as acetaminophen exposure during the first year of life. Medium exposure was defined as acetaminophen use once or more in the past year. High exposure was defined as acetaminophen use once or more per month in the past year. We included the largest sample from a single study if there were overlapping data between studies. For adjusted results, we included the data from the most adjusted model. Any disagreement was resolved by a third reviewer (J. Li). The authors were contacted if detailed information we required was not provided.

\section{Quality Assessment}

Two investigators assessed the risk of bias (Y. Gao and W. Cao). Discrepancies were resolved in discussion with a third investigator (J. Liu). The methodological quality of cohort and case-control studies was evaluated using the Newcastle-Ottawa scale (NOS) [22]. Each study was classified as low (scores of 0-3), medium (scores of 4-6), or high (scores of 7-9) quality. The methodological quality of cross-sectional studies was evaluated using a checklist recommended by the Agency for Healthcare Research and Quality (AHRQ) [23]. Each study was classified as low (scores of 0-3), medium (scores of 4-7), or high (scores of 8-11) quality.

\section{Statistical Analysis}

Statistical analysis was performed using Review Manager software (version 5.3) and Stata software (version 12.0). The ORs were used to assess the association between acetaminophen exposure and the risk of allergic rhinitis. The hazard ratios were considered as ORs. A random effect model was used considering the intertrial heterogeneity. Heterogeneity across studies was assessed using the $I^{2}$ index and Q statistic, where $I^{2}>50 \%$ indicated substantial heterogeneity and $p<0.1$ represented statistical significance [24]. Subgroup analyses were stratified by the age of participants, study design, study quality, location, sample size, and acetaminophen exposure. Sensitivity analysis was performed by removing one study at a time while keeping all other variables constant. Publication bias was evaluated by funnel plot analysis as well as Begg's test. A "trim-and-fill" procedure was conducted to assess the possible effect of publication bias [25].

\section{Results}

\section{Search Results}

A total of 1,678 references were identified in the initial search. After excluding duplicates and screening the ab- stracts and titles, we reviewed 48 full-text articles. We excluded 31 articles as they did not meet the inclusion criteria. Finally, 17 articles [13-20, 26-34] were included in the final analysis (Fig. 1).

\section{Study Characteristics}

A summary of the included studies is presented in Table 1 . The 17 articles [13-20, 26-34], including a total of 870,492 participants, were published from 2000 to 2018 . Among the 18 studies included in the 17 articles, 5 were cohort studies [18-20,28], 2 were case-control studies $[15,30]$, and 11 were cross-sectional studies $[13,14,16$, $17,26,27,29,31-34]$. Six studies were conducted in Europe $[15,17,20,27,32,34], 5$ studies in Asia [18, 29, 31, 33], 2 studies in Africa [16, 28], 2 studies in Oceania [19, 26], 1 study in South America [30], and 2 were multicountry studies $[13,14]$. Of the included studies, 6 were assessed as high quality $[13,14,18,20,33]$ and 12 as moderate quality $[15-17,19,26-32,34]$.

\section{Results of Meta-Analysis}

Acetaminophen exposure was associated with an increased risk of allergic rhinitis (OR: 1.54, 95\% CI:1.411.69 ) in 18 studies [13-20, 26-34] with 870,492 total participants (Fig. 2) (Table 2). Substantial heterogeneity was found among the studies $\left(I^{2}=94 \% ; p<0.00001\right)$. According to the sensitivity analysis, no single study affected the overall results or heterogeneity (Table 3 ).

For acetaminophen exposure in early life, the metaanalysis of 15 estimates from 11 studies $[13,17,18,20$, 26-29, 31-33] (510,796 participants) showed significant associations between acetaminophen exposure and allergic rhinitis (OR: 1.34, 95\% CI:1.21-1.49) (Fig. 2; Table 2). Substantial heterogeneity was found across the studies $\left(I^{2}=90 \% ; p<0.00001\right)$. In the sensitivity analysis, exclusion of any single study did not significantly affect the overall results or heterogeneity (Table 3 ).

For acetaminophen exposure in the past year, the meta-analysis of 20 estimates from 9 studies $[13,14,16,17$, $26,29,30,33,34](581,072$ participants) showed significant associations between acetaminophen exposure and allergic rhinitis (OR: 1.74, 95\% CI: 1.48-2.04) (Fig. 2; Table 2). Substantial heterogeneity was found across the studies $\left(I^{2}=96 \% ; p<0.00001\right)$. In the sensitivity analyses, no single study altered the overall results or heterogeneity (Table 3).

\section{Subgroup Analysis}

The results of the subgroup analysis are presented in Table 4. 


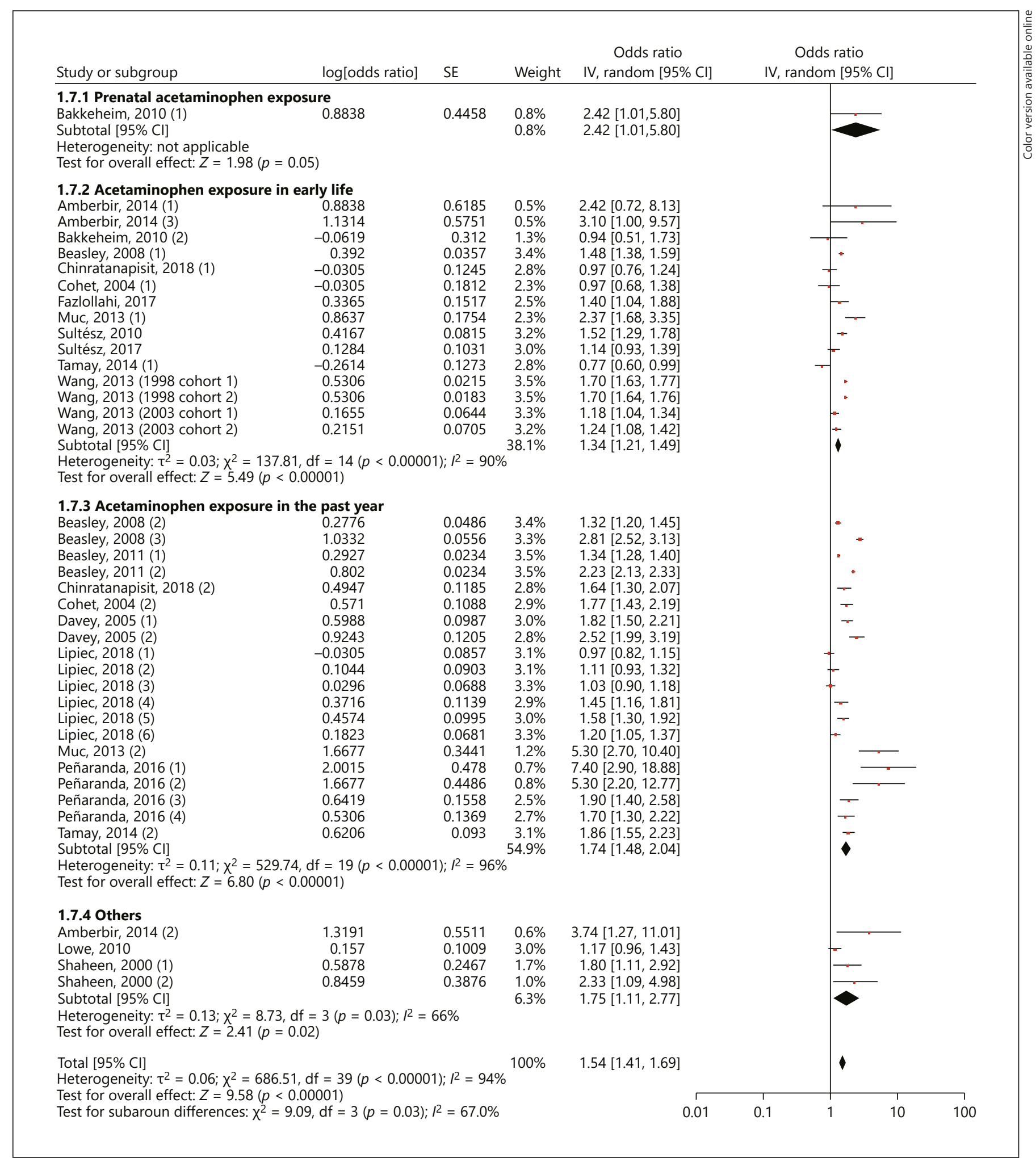

Fig. 2. Forest plot of the association between acetaminophen exposure and allergic rhinitis. CI, confidence interval.

Meta-Analysis of Acetaminophen and Allergic Rhinitis
Int Arch Allergy Immunol 2020;181:422-433 DOI: $10.1159 / 000506807$ 


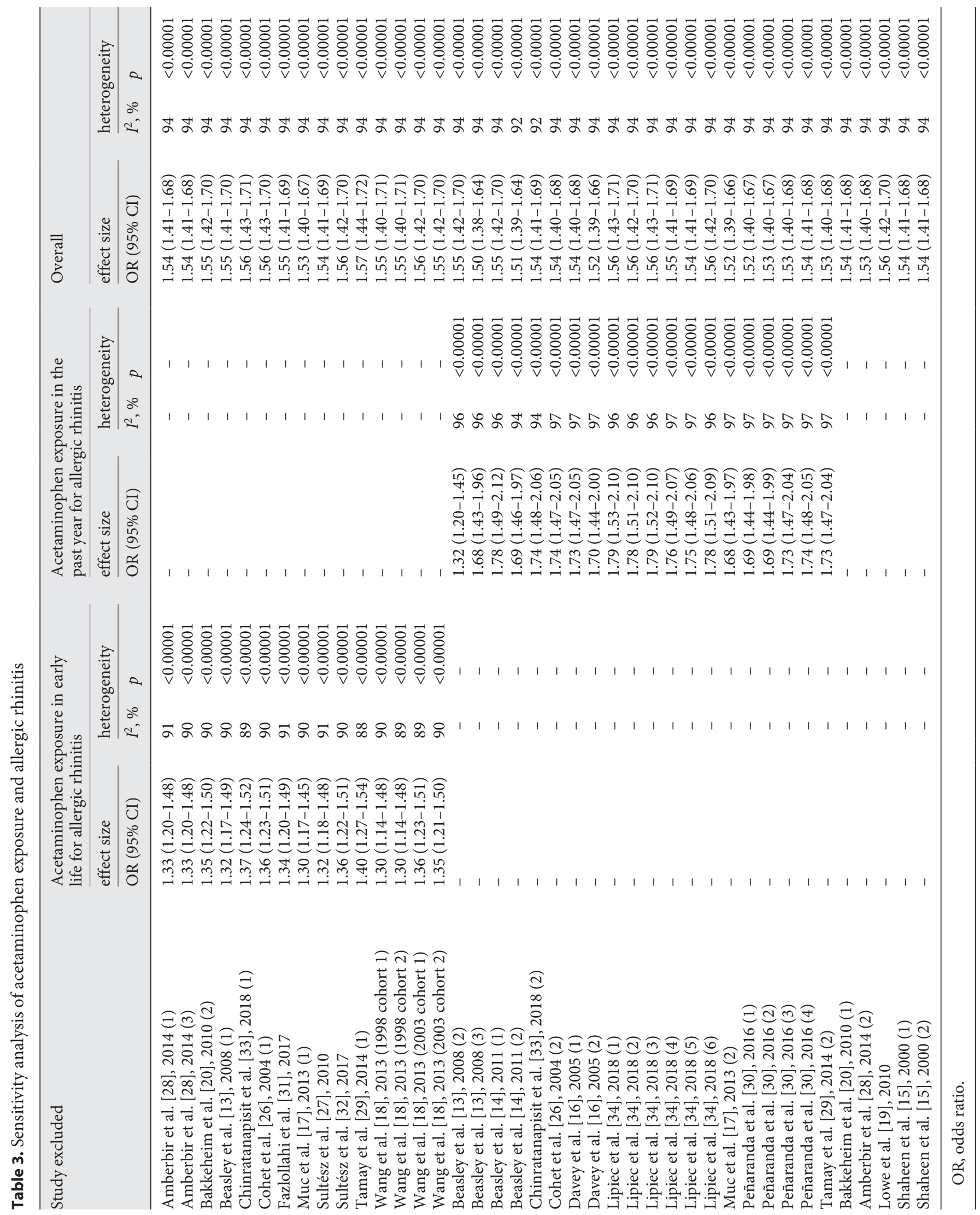




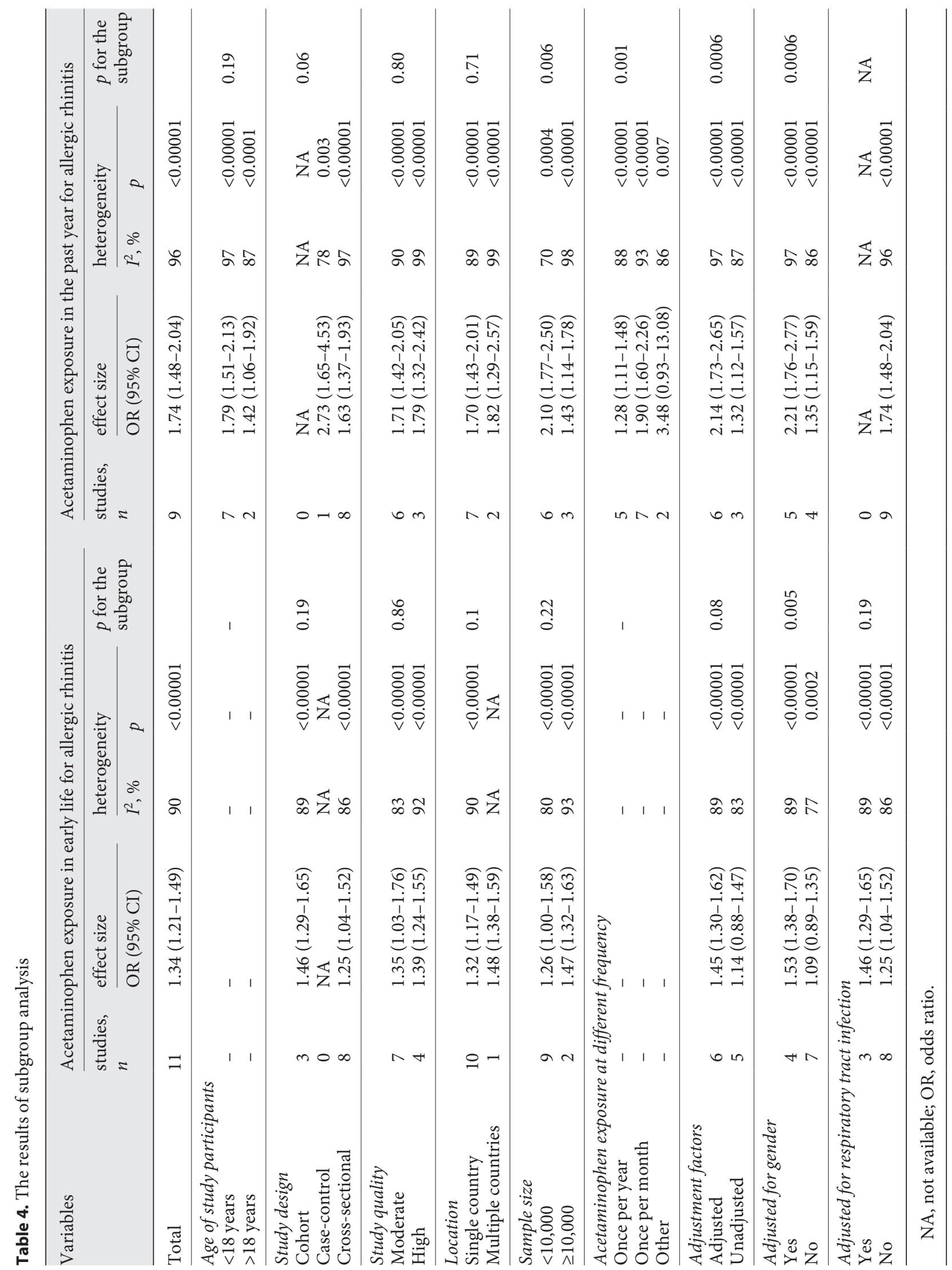


Acetaminophen Exposure in Early Life

Three cohort studies $[18,20,28]$ resulted in a pooled OR of 1.46 (1.29-1.65), whereas 8 cross-sectional studies $[13,17,26,27,29,31-33]$ had a pooled OR of $1.25(1.04-$ 1.52) $\left(\chi^{2}=1.74 ; p=0.19 ; I^{2}=42.4 \%\right)$. Four high-quality studies $[13,18,20,33]$ had a pooled OR of $1.39(1.24-$ $1.55)$, whereas 7 moderate-quality studies $[17,26-29,31$, 32] had a pooled OR of $1.35(1.03-1.76)\left(\chi^{2}=0.03 ; p=\right.$ $\left.0.86 ; I^{2}=0\right)$. The test for subgroup differences by adjustment for respiratory tract infection showed no significant differences $\left(\chi^{2}=1.74 ; p=0.19 ; I^{2}=42.4 \%\right)$. The 3 studies (7 estimates) $[18,20,28]$ adjusted for respiratory tract infection had a pooled OR of 1.46 (1.29-1.65), whereas 8 studies [13, 17, 26, 27, 29, 31-33] unadjusted for respiratory tract infection had a pooled OR of 1.25 (1.04-1.52). The test for subgroup differences with adjustment for confounding factors showed no significant differences $\left(\chi^{2}=2.97 ; p=0.08 ; I^{2}=66.3 \%\right)$. Six studies (10 estimates) $[13,17,18,20,28,33]$ adjusted for confounding factors had a pooled OR of 1.45 (1.30-1.62), whereas 5 studies $[26,27,29,31,32]$ unadjusted for confounding factors resulted in a pooled OR of $1.14(0.88-1.47)$. The test for subgroup differences by adjustment for gender indicated a significant difference $\left(\chi^{2}=7.99 ; p=0.005 ; I^{2}=87.5 \%\right)$. Four studies (8 estimates) $[13,17,18,28]$ adjusted for gender resulted in a pooled OR of $1.53(1.38-1.70)$, whereas 7 studies [20, 26, 27, 29, 31-33] unadjusted for gender resulted in a pooled OR of $1.09(0.89-1.35)$.

Acetaminophen Exposure in the Past Year

The test for subgroup differences by exposure frequency indicated a significant difference between once per month and once per year $\left(\chi^{2}=13.53 ; p=0.001 ; I^{2}=\right.$ $85.2 \%)$. People who were exposed to acetaminophen once per month in the past year $[13,14,16,26,29,30,34]$ (OR: $1.90,95 \%$ CI: 1.60-2.26) had a greater risk for allergic rhinitis compared with those who were exposed once per year [13, 14, 17, 33, 34] (OR: 1.28, 95\% CI: 1.11-1.48).

One case-control study [30] resulted in a pooled OR of 2.73 (1.65-4.53), whereas 8 cross-sectional studies [13, $14,16,17,26,29,33,34]$ had a pooled OR of 1.63 (1.371.93) $\left(\chi^{2}=3.61 ; p=0.06 ; I^{2}=72.3 \%\right)$. Three high-quality studies $[13,14,33]$ had a pooled OR of $1.79(1.32-2.42)$, whereas 6 moderate-quality studies $[16,17,26,29,30,34]$ resulted in a pooled OR of $1.71(1.42-2.05)\left(\chi^{2}=0.07 ; p=\right.$ $\left.0.80 ; I^{2}=0\right)$. The test for subgroup differences with adjustment for confounding factors showed significant differences $\left(\chi^{2}=11.92 ; p=0.0006 ; I^{2}=91.6 \%\right)$. Six studies $(12$ estimates) $[13,14,16,17,30,33]$ adjusted for confounding factors had a pooled OR of $2.14(1.73-2.65)$, whereas

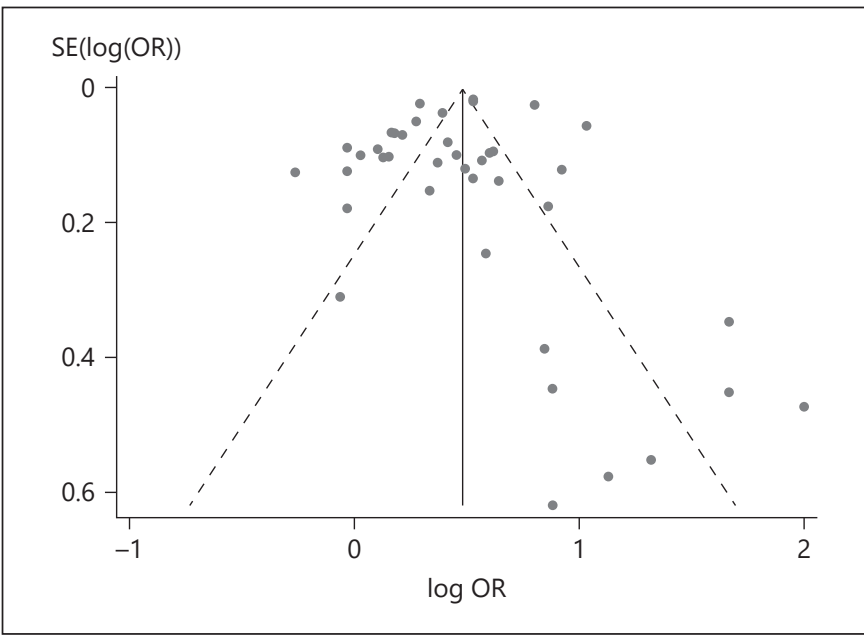

Fig. 3. Funnel plot of the association between acetaminophen exposure and allergic rhinitis. OR, odds ratio; SE, standard error.

3 studies (8 estimates) $[26,29,34]$ unadjusted for confounding factors had a pooled OR of 1.32 (1.12-1.57). The test for subgroup differences by adjustment for gender showed significant differences $\left(\chi^{2}=11.86 ; p=0.0006\right.$; $\left.I^{2}=91.6 \%\right)$. Five studies (11 estimates) $[13,14,16,17,30]$ adjusted for gender had a pooled OR of 2.21 (1.76-2.77), whereas 4 studies ( 9 estimates) $[26,29,33,34]$ unadjusted for gender had a pooled OR of 1.35 (1.15-1.59). The test for subgroup differences between participants below 18 years old $[13,14,16,17,26,29,33]$ (OR: 1.79, 95\% CI: $1.49-2.16)$ and participants above 18 years old [30, 34] (OR: 1.53, 95\% CI: 1.23-1.91) showed no significant differences $\left(\chi^{2}=1.74 ; p=0.19 ; I^{2}=42.5 \%\right)$.

\section{Publication Bias}

Results of the publication bias and trim-and-fill analysis are shown in Table 2. Visual inspection of the funnel plot showed a potential publication bias (Fig. 3; online suppl. eFig. 1, and 2; for all online supplementary material, see www.karger.com/doi/10.1159/000506807), although the result of the Begg's test showed no statistical significance (Table 3). We conducted the "trim-and-fill" analysis to estimate the number of missing studies that may have led to funnel plot asymmetry. As a result, one study on total acetaminophen exposure and 8 studies on acetaminophen exposure in early life, all with negative results, were found to be missing (Table 2). However, analysis with missing-value estimates did not produce different results (total acetaminophen exposure: OR 1.41 [95\% CI: 1.29-1.56]; acetaminophen exposure in early life: OR 1.33 [95\% CI: 1.20-1.48]). 


\section{Discussion}

In this meta-analysis based on 18 observational studies [13-20, 26-34] with 870,492 participants, we systematically evaluated the association between acetaminophen exposure and the risk of allergic rhinitis. The results reveal that acetaminophen exposure is associated with an increased risk of allergic rhinitis. In addition, acetaminophen exposure in early life is positively associated with the risk of allergic rhinitis, and individuals exposed to acetaminophen in the past year tend to have a higher risk of allergic rhinitis. Importantly, our results also show that there may be an exposure-dependent association between acetaminophen exposure in the past year and the risk of allergic rhinitis.

The current findings are biologically plausible. First, acetaminophen has been shown to reduce the levels of glutathione, an antioxidant present in the lung and respiratory mucosae $[35,36]$. The depletion of glutathione may increase the T-helper cell type 2 (TH2)-mediated immunological response found in allergic disorders [37]. Second, acetaminophen may influence cyclooxygenase- 2 activity and promote prostaglandin E2 synthesis and in turn, the TH2 response [38]. In addition, it has been demonstrated that chronic exposure to acetaminophen increases the mast cell infiltration of nasal mucosa in rat models [39].

Some potential confounding factors also need to be considered. For example, findings might be affected by respiratory infections, recall bias, and gender. Epidemiological studies have found an association between respiratory tract infection and risk of allergic rhinitis [40, 41]. Acetaminophen exposure for such episodes could cause confounding. Lowe et al. [19] reported that the association between acetaminophen use in the first 2 years of life and risk of allergic rhinitis vanished after adjustment for respiratory tract infections. However, a cohort study [18] of 263,620 children found acetaminophen use in early life was associated with an increased risk of allergic rhinitis in a group without respiratory infections. In our subgroup analysis, results showed a significant association between acetaminophen exposure in early life and the risk of allergic rhinitis after adjustment for respiratory tract infection. Therefore, early acetaminophen exposure might be a risk factor for allergic rhinitis.

Self-reports of acetaminophen use may be affected by recall bias. These limitations are addressed by prospective cohort study designs, which reduces recall bias. In our subgroup analysis, the findings from the prospective cohort studies subgroup indicated a significant association

Meta-Analysis of Acetaminophen and Allergic Rhinitis between acetaminophen exposure in early life and the risk of allergic rhinitis. Gender may also affect the risk of allergic rhinitis $[42,43]$. In our subgroup analysis, the association between acetaminophen exposure and allergic rhinitis was even stronger when adjusted for gender.

Several factors suggest that there is a positive association between acetaminophen exposure and allergic rhinitis. First, our study showed an exposure-dependent association between acetaminophen exposure in the past year and the risk of allergic rhinitis. Second, acetaminophen exposure in early life and acetaminophen exposure later in childhood were associated with an increased risk of allergic rhinitis. Third, according to subgroup analysis, there was a consistent association between acetaminophen exposure and allergic rhinitis in groups of participants below 18 years old and above 18 years old. Finally, per capita acetaminophen sales have been positively associated with the prevalence of allergic rhinitis in children and adults [12].

Our study has several strengths. First, this metaanalysis evaluated the association between acetaminophen exposure and allergic rhinitis using the recommended guidelines to ensure rigor. Second, our analysis included 870,492 participants from 18 studies. The study was multinational with a wide range of geographical representation. Third, by using the frequency of acetaminophen exposure as a categorical variable, we found an exposure-dependent association with the risk of allergic rhinitis. Fourth, the pooled risk remained consistent in the sensitivity analysis, suggesting that the results were stable.

The limitations of our study also need to be considered. First, some diagnosis of allergic rhinitis was based on parental questionnaires without objective clinical examinations. Second, self-report of acetaminophen use may be subject to recall bias. Third, the substantial heterogeneity indicates that methodological diversity existed among the included studies. Finally, the residual confounding factors without being fully adjusted may confuse the results. However, the associations were still significant when controlled for confounding factors in subgroup analysis.

In this meta-analysis, we evaluated the relationship between acetaminophen exposure and allergic rhinitis. There was only one study that focused on the relationship between maternal acetaminophen exposure and allergic rhinitis in offspring, which limited our ability to analyze this subgroup. More high-quality research is needed in this area. In addition, there were no studies that assessed the association between acetaminophen exposure and 
objective atopy measurements, such as allergen-specific immunoglobulin E levels or skin prick testing. Finally, causality cannot be established based on the present results. Further studies, in which confounders are fully adjusted and objective biological assessment, are needed to explore the exact relationship between acetaminophen exposure and allergic rhinitis to establish whether the association is causal.

\section{Conclusions}

This study provides evidence that exposure to acetaminophen is associated with an increased risk of allergic rhinitis. In addition, acetaminophen exposure in early life and acetaminophen exposure in the past year are positively associated with the risk of allergic rhinitis. Future research is needed to evaluate whether the association is causal.

\section{Disclosure Statement}

The authors have no conflicts of interest to declare.

\section{Funding Sources}

This work was supported by the Science and Technology Project of the Health Planning Committee of Sichuan (17PJ066), the Grants from the Project of Sichuan Medical Association (2018SHD4-4), the Grants from the Youth Innovation Project of Sichuan Medical Research (Q16029), the Grants from Science and Technology Bureau of Deyang City (2015SZ063).

\section{Author Contributions}

Drs Song and Zeng conceptualized and designed the study, drafted the initial manuscript, and reviewed and revised the manuscript. Drs Gao, Cao, and Qianfang Liu designed the data collection form, collected data, and carried out the initial analyses. Drs Li and Jian Liu coordinated and supervised data collection. All authors reviewed the final manuscript as submitted and agree to be accountable for all aspects of the work.

\section{References}

1 Settipane RA. Demographics and epidemiology of allergic and nonallergic rhinitis. Allergy Asthma Proc. 2001 Jul-Aug;22(4):185-9.

2 Singh K, Axelrod S, Bielory L. The epidemiology of ocular and nasal allergy in the United States, 1988-1994. J Allergy Clin Immunol. 2010 Oct;126(4):778-783.e6.

3 Wu WF, Wan KS, Wang SJ, Yang W, Liu WL. Prevalence, severity, and time trends of allergic conditions in 6-to-7-year-old schoolchildren in Taipei. J Investig Allergol Clin Immunol. 2011;21(7):556-62.

4 Meltzer EO, Blaiss MS, Derebery MJ, Mahr TA, Gordon BR, Sheth KK, et al. Burden of allergic rhinitis: results from the Pediatric Allergies in America survey. J Allergy Clin Immunol. 2009 Sep;124(3 Suppl):S43-70.

5 Abdulrahman H, Hadi U, Tarraf H, Gharagozlou M, Kamel M, Soliman A, et al. Nasal allergies in the Middle Eastern population: results from the "Allergies in Middle East Survey". Am J Rhinol Allergy. 2012 Nov-Dec; 26(6_suppl Suppl 1):S3-23.

6 Torrance GW. Preferences for health outcomes and cost-utility analysis. Am J Manag Care. 1997 May;3 Suppl:S8-20.

7 Ray NF, Baraniuk JN, Thamer M, Rinehart CS, Gergen PJ, Kaliner M, et al. Direct expenditures for the treatment of allergic rhinoconjunctivitis in 1996, including the contributions of related airway illnesses. J Allergy Clin Immunol. 1999 Mar;103(3 Pt 1):401-7.

8 Bender BG. Cognitive effects of allergic rhinitis and its treatment [vi-vii.]. Immunol Allergy Clin North Am. 2005 May;25(2):301-12.
9 Ferreira MA, Vonk JM, Baurecht H, Marenholz I, Tian C, Hoffman JD, et al.; 23andMe Research Team; AAGC collaborators; BIOS consortium; LifeLines Cohort Study. Shared genetic origin of asthma, hay fever and eczema elucidates allergic disease biology. Nat Genet. 2017 Dec;49(12):1752-7.

10 Galassi C, De Sario M, Biggeri A, Bisanti L, Chellini E, Ciccone G, et al. Changes in prevalence of asthma and allergies among children and adolescents in Italy: 1994-2002. Pediatrics. 2006 Jan;117(1):34-42.

11 Bunchorntavakul C, Reddy KR. Acetaminophen-related hepatotoxicity [viii.]. Clin Liver Dis. 2013 Nov; 17(4):587-607.

12 Newson RB, Shaheen SO, Chinn S, Burney PG. Paracetamol sales and atopic disease in children and adults: an ecological analysis. Eur Respir J. 2000 Nov;16(5):817-23.

13 Beasley R, Clayton T, Crane J, von Mutius E, Lai CK, Montefort S, et al.; ISAAC Phase Three Study Group. Association between paracetamol use in infancy and childhood, and risk of asthma, rhinoconjunctivitis, and eczema in children aged 6-7 years: analysis from Phase Three of the ISAAC programme. Lancet. 2008 Sep;372(9643):1039-48.

14 Beasley RW, Clayton TO, Crane J, Lai CK, Montefort SR, Mutius E, et al.; ISAAC Phase Three Study Group. Acetaminophen use and risk of asthma, rhinoconjunctivitis, and eczema in adolescents: International Study of Asthma and Allergies in Childhood Phase Three. Am J Respir Crit Care Med. 2011 Jan; 183(2):171-8.
15 Shaheen SO, Sterne JA, Songhurst CE, Burney PG. Frequent paracetamol use and asthma in adults. Thorax. 2000 Apr;55(4):266-70.

16 Davey G, Berhane Y, Duncan P, Aref-Adib G, Britton J, Venn A. Use of acetaminophen and the risk of self-reported allergic symptoms and skin sensitization in Butajira, Ethiopia. J Allergy Clin Immunol. 2005 Oct;116(4):8638.

17 Muc M, Padez C, Pinto AM. Exposure to Paracetamol and Antibiotics in Early Life and Elevated Risk of Asthma in Childhood. Adv Exp Med Biol. 2013;788:393-400.

18 Wang JY, Liu LF, Chen CY, Huang YW, Hsiung CA, Tsai HJ. Acetaminophen and/or antibiotic use in early life and the development of childhood allergic diseases. Int J Epidemiol. 2013 Aug;42(4):1087-99.

19 Lowe AJ, Carlin JB, Bennett CM, Hosking CS Allen KJ, Robertson CF, et al. Paracetamol use in early life and asthma: prospective birth cohort study. BMJ. 2010 Sep;341 sep15 1:c4616.

20 Bakkeheim E, Mowinckel P, Carlsen KH, Håland G, Carlsen KC. Paracetamol in early infancy: the risk of childhood allergy and asthma. Acta Paediatr. 2011 Jan;100(1):90-6.

21 Stroup DF, Berlin JA, Morton SC, Olkin I, Williamson GD, Rennie D, et al. Meta-analysis of observational studies in epidemiology: a proposal for reporting. Meta-analysis Of Observational Studies in Epidemiology (MOOSE) group. JAMA. 2000 Apr;283(15): 2008-12.

22 Stang A. Critical evaluation of the NewcastleOttawa scale for the assessment of the quality of nonrandomized studies in meta-analyses. Eur J Epidemiol. 2010 Sep;25(9):603-5. 
23 Rostom A, Dube C, Cranney A, et al. Celiac Disease. Rockville (MD): Agency for Healthcare Research and Quality (US); 2004 Sep (Evidence Reports/Technology Assessments, No 104). Appendix D. Quality Assessment Forms. Available from: http://www.ncbi.nlm. nih.gov/books/NBK35156.

24 Higgins JP, Thompson SG, Deeks JJ, Altman DG. Measuring inconsistency in meta-analyses. BMJ. 2003 Sep;327(7414):557-60.

25 Duval S, Tweedie R. Trim and fill: A simple funnel-plot-based method of testing and adjusting for publication bias in meta-analysis. Biometrics. 2000 Jun;56(2):455-63.

26 Cohet C, Cheng S, MacDonald C, Baker M, Foliaki S, Huntington N, et al. Infections, medication use, and the prevalence of symptoms of asthma, rhinitis, and eczema in childhood. J Epidemiol Community Health. 2004 Oct;58(10):852-7.

27 Sultész M, Katona G, Hirschberg A, Gálffy G. Prevalence and risk factors for allergic rhinitis in primary schoolchildren in Budapest. Int J Pediatr Otorhinolaryngol. 2010 May;74(5) 503-9.

28 Amberbir A, Medhin G, Hanlon C, Britton J, Davey G, Venn A. Effects of early life paracetamol use on the incidence of allergic disease and sensitization: 5 year follow-up of an Ethiopian birth cohort. PLoS One. 2014 Apr;9(4):e93869.

29 Tamay Z, Akçay A, Ergin A, Güler N. Prevalence of allergic rhinitis and risk factors in 6to 7-year-old children in İstanbul, Turkey. Turk J Pediatr. 2014 Jan-Feb;56(1):31-40.
30 Peñaranda A, Garcia E, Barragán AM, Rondón MA, Pérez A, Rojas MX, et al. Factors associated with Allergic Rhinitis in Colombian subpopulations aged 1 to 17 and 18 to 59 . Rhinology. 2016 Mar;54(1):56-67.

31 Fazlollahi MR, Abbasi JM, Dana VG, Yousefzade A, Sabetkish N, Haghighat S. Evaluation of Allergic Symptoms Prevalence and Its Relationship with Acetaminophen/Antibiotic Use and Hospitalization Among School-Aged Children in Tehran, Iran. Iran Red Crescent Med J. 2017;19(5):e45724. https://doi. org/10.5812/ircmj.45724.

32 Sultész M, Balogh I, Katona G, Mezei G, Hirschberg A, Gálffy G. Trends in prevalence and risk factors of allergic rhinitis symptoms in primary schoolchildren six years apart in Budapest. Allergol Immunopathol (Madr). 2017 Sep-Oct;45(5):487-95.

33 Chinratanapisit S, Suratannon N, Pacharn P, Sritipsukho P, Vichyanond P. Prevalence and risk factors of allergic rhinitis in children in Bangkok area. Asian Pac J Allergy Immunol. 2019 Dec;37(4):232-9.

34 Lipiec A, Wawrzyniak ZM, Sybilski AJ, Samolińska-Zawisza U, Krzych-Fałta E, Piekarska B, et al. The association between paracetamol use and the risk of asthma, rhinitis and eczema in the Polish population. Ann Agric Environ Med. 2018 Sep;25(3):428-32.

35 Kelly FJ. Gluthathione: in defence of the lung. Food Chem Toxicol. 1999 Sep-Oct;37(9-10): 963-6.

36 Nuttall SL, Khan JN, Thorpe GH, Langford N, Kendall MJ. The impact of therapeutic doses of paracetamol on serum total antioxidant capacity. J Clin Pharm Ther. 2003 Aug;28(4): 289-94.
37 Peterson JD, Herzenberg LA, Vasquez K, Waltenbaugh C. Glutathione levels in antigen-presenting cells modulate Th1 versus Th2 response patterns. Proc Natl Acad Sci USA. 1998 Mar;95(6):3071-6.

38 Swierkosz TA, Jordan L, McBride M, McGough K, Devlin J, Botting RM. Actions of paracetamol on cyclooxygenases in tissue and cell homogenates of mouse and rabbit. Med Sci Monit. 2002 Dec;8(12):BR496-503.

39 Caballero N, Welch KC, Carpenter PS, Mehrotra S, O'Connell TF, Foecking EM. Association between chronic acetaminophen exposure and allergic rhinitis in a rat model. $\mathrm{Al}-$ lergy Rhinol (Providence). 2015 Jan;6(3): $162-7$.

40 Ciprandi G, Tosca MA, Fasce L. Allergic children have more numerous and severe respiratory infections than non-allergic children. $\mathrm{Pe}$ diatr Allergy Immunol. 2006 Aug;17(5):38991.

41 de Oliveira TB, Klering EA, da Veiga AB. Is recurrent respiratory infection associated with allergic respiratory disease? J Asthma. 2019 Feb;56(2):160-6.

42 Keller T, Hohmann C, Standl M, Wijga AH, Gehring U, Melén E, et al. The sex-shift in single disease and multimorbid asthma and rhinitis during puberty - a study by MeDALL. Allergy. 2018 Mar;73(3):602-14.

43 Pinart M, Keller T, Reich A, Fröhlich M, Cabieses B, Hohmann C, et al. Sex-Related Allergic Rhinitis Prevalence Switch from Childhood to Adulthood: A Systematic Review and Meta-Analysis. Int Arch Allergy Immunol. 2017;172(4):224-35. 